cannot be ionized in the native protein, the appearance of an extrinsic Cotton effect associated with the aromatic side chains and the low intrinsic viscosity, leave no reasonable doubt that the enzyme at room temperature is in a specific, folded globular conformation. The enzyme, of course, functions at high temperature, and when kept at $80^{\circ} \mathrm{C}$ for one hour and then cooled shows an undiminished maximum velocity and only slightly increased Michaelis constant. It is particularly remarkable that the conformation is completely unaffected by $8 \mathrm{M}$ urea, usually regarded as an essentially universal denaturant. It is found, however, that after prolonged heat treatment conformational changes have occurred: the anomalous tyrosine residues appear to have become normalized, and the helix content is diminished.

The full significance of these observations and their relation to the conformational stability in vivo are not yet clear. Neither has the present work yet answered the question of whether in fact the conformation remains intact at the high temperature or whether the molecule exists in a wholly or partially unfolded state which reverts in the presence of the substrate. It is worth noting that the stabilization of a native enzyme structure by substrate-antibody by hapten, for examplehas been frequently reported. Indeed, it is thermodynamically obvious that if a substrate binds only (or more strongly) to the native conformation, the latter must be correspondingly stabilized with respect to the native state when binding occurs.

The source of the unusual stability of these enzymes remains obscure, though Ohta has noted that the protein contains a rather high proportion of hydrophobic residues. It may be recalled that the constituents of the protein synthesis system of a thermophile were recently studied in an interesting series of papers by a group of Italian workers, and that the amino-acid-sRNA synthetases, a notoriously unstable and intractable class of enzymes, turned out to function in vitro at temperatures above $70^{\circ} \mathrm{C}$ in $3 \mathrm{M}$ urea. The system was used to examine the effect of the denaturation of $s$ RNA on its activity, and no doubt further interesting applications in relation to the conformation of various substrate species will be found for enzymes from these organisms. It is also to be hoped that some of the more complex enzymes, especially those with quaternary structure, will in time be examined.

\section{Bacterial Ancestry}

\section{from a Correspondent in Microbiology}

ORTHODOx classifications of bacteria are erected on phenotypic data, usually very few and consequently representing but a small portion of the total genome. Classifications based on numerical analysis are superior in that 10-20 per cent of the genome is sampled when 200 or so characters are considered. The classification may be improved further by examining the extent of DNA identity using such parameters as homology and base composition, and this approach has been exploited brilliantly in recent years by $J$. do Ley and his associates at the State University in Ghent. The latest report from this laboratory discusses relationships among ccrtain pseudomonads and xanthomonads and presents some fascinating clues to the phylogeny of this group of bacteria (Park, I. W., and de Ley, J., Antonie van Leeuwenhoek, 33, 1; 1967).
Reciprocal hybridizations were made with DNA from Pseudomonas fluorescens, Pseudomonas putida and the xanthomonad Pseudomonas campestris var. pelargonii; and between DNA fragments from each strain, preselected by hybridization with each of the other two strains, and all three "whole" DNA types. The results from these experiments indicate that a certain part of each of the three genomes is similar and is designated the "common" or "fluorescens-putida-pelargonii" part. Further, the fluorescens and putida strains are more closely related to each other than to pelargonii, while the latter has greater affinity to fluorescens than to putida. The molecular weights of the DNA from the three strains are closely similar $\left(2.4 \pm 0.4 \times 10^{9}\right)$ with a value suggesting the accommodation of about 3,900 cistrons. The "common" part, accounting for about 50 per cent of the genome of each strain, must be that coding for the shared phenotypic attributes and including cell morphology, aerobiosis, polar flagellation and common enzymes such as catalase and the EntnerDoudoroff enzymes.

The fluorescens-putida common part, amounting to about 33 per cent of the respective genomes, will accommodate 1,300 cistrons controlling characters such as the green pigmentation, enzymes for the oxidation of gluconate, utilization of nitrite, ammonia and urea and many particulate enzymes. Species differentiation between $P$. fluorescens and $P$. putida is based on the remaining 17 per cent or approximately 650 cistrons. The authors propose that the 50 per cent piece of pelargonii-specific DNA might consist of two components: (i) a component common to all xanthomonads, and (ii) a component typical of the strain pelargonii. The general xanthomonad part probably codes at least for carotenoid and polysaccharide syntheses and phytopathogenicity. (See de Ley, J., et al., J. Gen. Microbiol., 42, 43; 1966, for further data.)

These data lead Park and de Ley to suggest that the three bacteria arose from a pool of common ancestors and that half of each genome is still retained and related very closely to the original ancestral genome. Accordingly, the ancestor may be visualized as an aerobic, polar flagellate bacillus possessing Entner-Doudoroff and shunt pathways, catalase and the capacity to oxidize a variety of carbohydrates. Moreover, we may reasonably conclude that the xanthomonad diverged first or most quickly from such a stock and that the discontinuity between fluorescens and putida was of more rcsent origin. Consideration of data in de Ley et al. allows a more comprehensive reconstruction of pseudomonad phylogeny. Arguing from the phytopathogenicity of the xanthomonads, Park and de Ley presume that the latter only arose after the appearance of angiosperms about 135 million years ago, The "common" part of the genome, comprising some 2 million base pairs, indicates the comparable antiquity of the pseudomonads. Tho average mutation rate in this "common" part is considered to be less than 1 base pair per 700 years, while that of the dissimilar part of the genome would be 1 to 10 , or more, base pairs per 700 ycars, depending on when the xanthomonads arose during the 135 million year span. At last, therefore, we have some objective comment on bacterial evolution, however slight, to replace previous speculations. Moreover, the extension of such analyses should lead to the greatest possible precision in bacterial classification. 